\title{
Light-harvesting positively-charged nanocrystals for strong energy transfer to dye molecules
}

\section{Evren Mutlugun and Hilmi Volkan Demir}

Department of Physics, Department of Electrical and Electronics Engineering, Nanotechnology Research Center, and Institute of Materials Science and Nanotechnology, Bilkent University, Ankara, 06800 Turkey

Dye molecules find several device applications including dye based lasers [1]. Because of their high quantum efficiency, such dye molecules are beneficial for use in optoelectronic devices to yield high efficiency. However, dye molecules typically exhibit quite narrow spectral excitation ranges where they can be efficiently pumped, limiting their use in certain optoelectronic applications that require wider spectral response. For example, rhodamine B molecules have emission at $580 \mathrm{~nm}$ and they can be pumped optimally only at around $550 \mathrm{~nm}$, corresponding to their absorption maximum. But, when going outside this spectral excitation range, it quickly becomes very inefficient to pump rhodamine B molecules.

Semiconductor nanocrystal quantum dots, on the other hand, feature size-tuneable optical emission, absorption, and excitation characteristics, which can be conveniently tuned and optimized in principle in accordance with specific application. As a result of their wide-range tunability, nanocrystals find numerous device applications, e.g., in UV [2]. Nanocrystals can also be used as light harvesters for energy transfer to other luminescent molecules [3]. In this work, we present water-soluble positively-charged $\mathrm{CdSe} / \mathrm{ZnS}$ core/shell nanocrystals serving as light harvester (donor) for strong energy transfer to rhodamine B dye molecules (acceptor) in solution. By doing so, rhodamine molecules can be effectively pumped through energy transfer at shorter wavelengths beyond its excitation spectrum.

Because of the short-range energy transfer, by choosing appropriate spacing, sizes, and charges of the interacting particles, it is possible to enhance energy transfer. To this end, we used positively charged nanocrystal emitters because rhodamine $\mathrm{B}$ forms an acidic solution, releasing protons and being left effectively negative. From the spectral point, we designed nanocrystals to emit at $540 \mathrm{~nm}$ to match rhodamine B molecules. Also, utilizing Coulomb attraction between positively charged nanocrystals and negative rhodamine B molecules to diminish the spacing between them in solution, we achieved strong transfer energy.

To enhance the emission of rhodamine molecules, we systematically employed different blends of rhodamine Bnanocrystal mixtures in controlled amounts. We performed time resolved measurements on these controlled blends starting with $1.20 \mathrm{nmol}$ of $\mathrm{CdSe} / \mathrm{ZnS}$ core/shell nanocrystals and successively increasing the amount of rhodamine B molecules. The lifetime measurements were performed using PicoQuant with a laser head at $374 \mathrm{~nm}$. Figure 1 shows the quenching of the nanocrystal emitters with increasing amount of rhodamine B molecules. This measurements show that the nanocrystals efficiently transfer energy to dye molecules and consequently quench, with their lifetimes decreasing successively, and reaching an optimal level of energy transfer at $23.254 \mathrm{nmol}$ of rhodamine B molecules per $1.20 \mathrm{nmol}$ of $\mathrm{CdSe} / \mathrm{ZnS}$ nanocrystals. The time constants for each case are also presented in Table 1.

Furthermore, we performed the steady state photoluminescence measurements of the best performing combination of nanocrystals and dye molecules. Figure 2 shows that the nanocrystals transfer energy to rhodamine B molecules and enhance their emission by $35 \%$ at the laser pump wavelength at the steady state. These results are in good agreement with the time resolved fluorescence data.

In conclusion, as a proof-of-concept demonstration, we showed light-harvesting nanocrystals used as highly efficient donor molecules for energy transfer to acceptor dye molecules. In accordance with PL characterization, we enhanced the emission of rhodamine B by $35 \%$ at the laser pump wavelength, which may open up possibilities for lasers based on dye molecules pumped at different wavelengths.

Acknowledgements: This work is supported by EU MOON, EU NOE PHOREMOST and TUBITAK 104E114, 106E020, 107E088, 107E297, 105E065, and 105E066. H.V.D. also acknowledges support from TUBA GEBIP and ESF-EURYI

\section{References}

[1I I. Kolbin et. al, "Thin-film rhodamine B laser with a distributed feedback”, Sov. J. Quantum Electron. 5, 860-861 (1975)

[2] E. Mutlugun et. al, "Nanocrystal hybridized scintillators for enhanced detection and imaging on Si platforms in UV," Opt. Express 15, 11281134 (2007).

[3] E. Alphandery et. al, "Highly efficient F€orster resonance energy transfer between CdTe nanocrystals and Rhodamine B in mixed solid films" Chemical Physics Letters 388, 100-104(2004) 


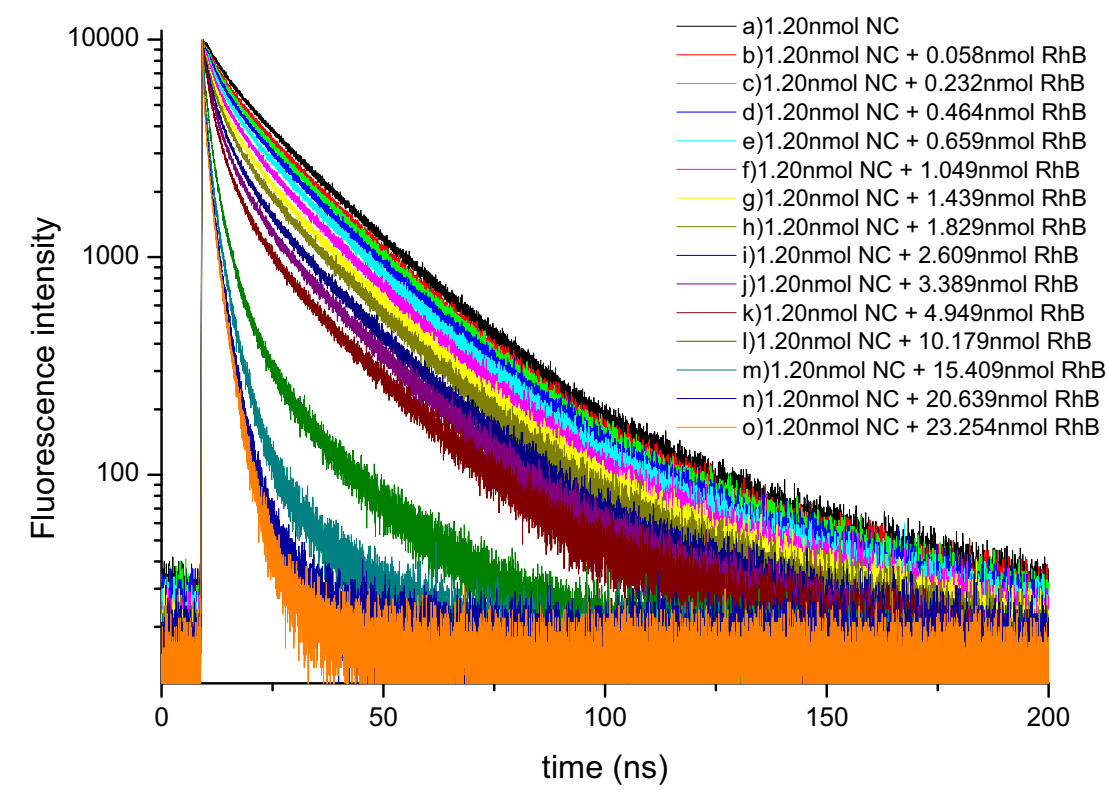

Figure 1. Fluorescence decay curves of nanocrystals (donors) parameterized with respect to the increasing amount of rhodamine $B$ molecules (acceptors) at the peak emission wavelength of the nanocrystals.

Table 1. Fluorescence decay times of nanocrystals (donors) as a function of increasing amount of dye molecule s (acceptors). [The letters a-o indicate the same set as given in Fig.1.]

\begin{tabular}{|l|r|r|r|r|r|r|r|r|r|r|r|r|r|r|r|}
\hline Time (ns) & $\mathrm{a}$ & $\mathrm{b}$ & $\mathrm{c}$ & $\mathrm{d}$ & $\mathrm{e}$ & $\mathrm{f}$ & $\mathrm{g}$ & $\mathrm{h}$ & $\mathrm{i}$ & $\mathrm{j}$ & $\mathrm{k}$ & $\mathrm{l}$ & $\mathrm{m}$ & $\mathrm{n}$ & $\mathrm{o}$ \\
\hline $\mathrm{t} 1 \mathrm{1}$ & 33.67 & 32.55 & 30.16 & 30.4 & 25.05 & 24.49 & 23.46 & 22.84 & 22.47 & 22.21 & 21.67 & 18.88 & 12.51 & 4.6 & 3.78 \\
\hline $\mathrm{t} 2$ & 17.62 & 16.6 & 15.53 & 15.49 & 10.16 & 9.04 & 7 & 5.95 & 5.46 & 5.07 & 4.55 & 3.87 & 2.66 & 1.65 & 1.35 \\
\hline $\mathrm{t} 3$ & 2.41 & 2.04 & 2.15 & 2.311 & 1.542 & 1.46 & 1.08 & 0.88 & 0.84 & 0.72 & 0.68 & 0.52 & 0.36 & 0.2 & 0.19 \\
\hline
\end{tabular}

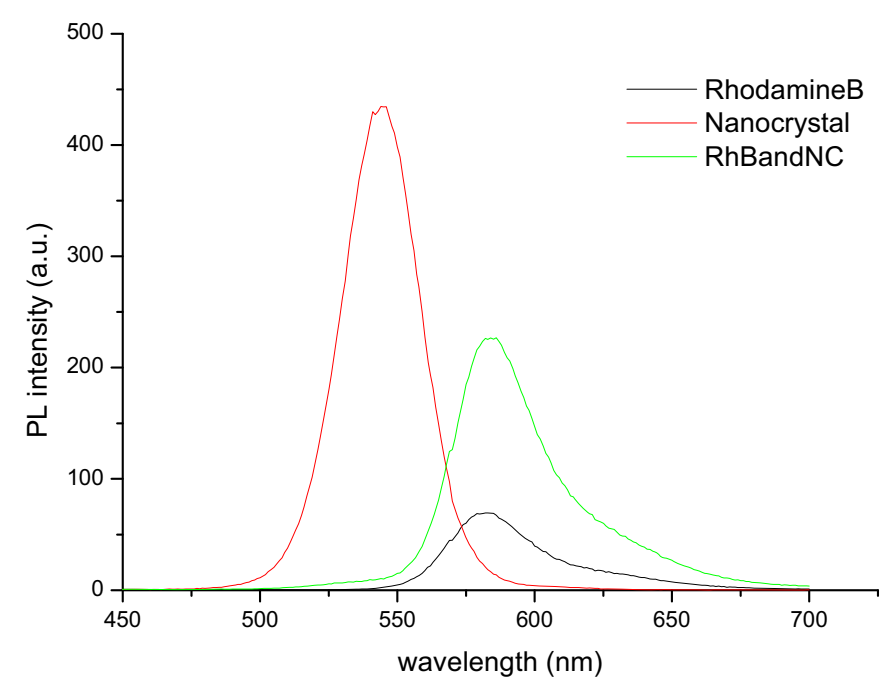

Figure 2. Steady state photoluminescence measurements of the nanocrystals, the dye molecules, and their mixed blends. 